\title{
The Effects of Music during Balance Training for Six Weeks on Elderly Persons' Balancing Ability
}

\begin{abstract}
The purpose of the present study is to provide exercises, together with music, that are helpful for elderly people's balancing ability, and to determine their effects in order to provide data for the promotion of elderly persons' health. Thirty elderly persons were randomly assigned to a balance training group(BTG) of 15 subjects, or a music and balance training group (music therapy + balance training [MTBTG]) of 15 subjects; intervention was implemented three times per week for six weeks. To measure the changes in their balancing ability before and after the experiment, the limit of stability, the "Timed Up and Go" (TUG) test, and the Berg Balance Scale (BBS) were measured. Changes in the limit of stability before and after the experiment were shown to be significant in both the BTG and the MTBTG. Changes in the limit of stability of the right side before and after the experiment showed statistically significant differences between the BTG and the MTBTG. Changes in the TUG test and the BBS before and after the experiment were shown to be statistically significant in both the BTG and the MTBTG. The application of music during balance training thus is considered to have a positive effect on elderly persons' balancing ability.
\end{abstract}

Key words: Music Therapy; Balance Training; Elderly

\begin{abstract}
Nyeon Jun Kimª, Jung Hyun Choi ${ }^{\text {, }}$ Ho Jung $\mathrm{An}^{\mathrm{c}}$, Ji Sung $\mathrm{Kim}^{\mathrm{d}}$, Yoo Rim Choie, Jung San Wang', Sang Bin Lee ${ }^{b}$, Kyung Tae Yoo ${ }^{b}$, Hyun Sook Hwang ${ }^{g}$, Soon Hee $\mathrm{Kim}^{\mathrm{h}}$.

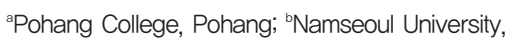
Cheonan; 'Dongnam Health University, Suwon; 'Suwon Women's College, Suwon; 'Taegu Science University, Daegu; 'Yeoju Institute of Technology, Yeoju; ' $\mathrm{C}$ Cheju Halla University, Jeju; "Yongin University, Yongin.
\end{abstract}

Received: 3 Nomember 2014

Revised : 21 January 2015

Accepted : 7 February 2015

Address for correspondence

Soon Hee Kim, PT, Ph.D

Department of Physical Therapy, Yongin University, 470 Samga-dong, Cheoingu, Yongin, Korea

Tel: 82-31-8020-2774

E-mail: shkim@yongin.ac.kr

\section{INTRODUCTION}

The most remarkable changes that appear in elderly persons are changes in their muscles and skeletal systems. As aging progresses, elderly persons begin to complain of muscle weakness and fatigue, and the functions of their musculoskeletal system(among other systems) start to decrease as their motility decreases along with decreases in the ability of the muscles that maintain posture. Consequently, geriatric persons' motor abilities decrease, which have direct effects on the quality of their daily lives(1).

The aging process leads to decreases in musculature and balancing ability, both of which increase the risk of falls(2). Although the mechanisms that lead to these decreases in balancing ability are complicated, one of the most notable features in the aging process is the decrease in musculature. As aging progresses, the fat and collagen in muscles increases, thus leading to a decrease in muscle strength(3,4). In addition, along with the decrease in physical strength due to the aging process, muscles atrophy. Elderly persons' muscle weakening affects their maintenance of balance; weakening of the lower extremity muscles, in particular, has been reported as a leading cause of falls $(5,6)$. Although these declines in physical strength during senescence cannot be completely prevented, the rates of decrease in physical strength can be reduced, and physical strength can be increased by appropriate physical activities and regular exercise. Physical therapists should therefore select several important elements of physical strength, and elderly people should perform exercises that can strengthen these elements(7). 
Elderly persons' exercise is effective in combating the muscular atrophy, loss of self-esteem, and depression that accompany ill-health during senescence(8). In addition, researchers have reported that exercise strengthens elderly persons' bones, and enhances their flexibility as well as overall cardiovascular system functions and body balancing ability $(9,10)$. Therefore, elderly people are actively encouraged to exercise. The exercise intensity should be maintained within a range of 40 85 percent of an individual's maximum exercise ability(11). The frequency of exercise is recommended to be $3 \sim 5$ times per week for $30 \sim 40$ minutes per session; the time to see the effects of exercise should be 8 12 weeks(12).

Many studies have been conducted on different methods for preventing damage to the elderly during exercise or daily living activities, as well as preserving their physical ability. As a non-pharmacological approach, many researchers have discussed the benefits of music therapy. One report has indicated that compared to exercising without music, elderly persons showed more fluid movements when they exercised while listening to music 13); some researchers have reported that programs that combine music and movements for elderly persons with dementia help daily living activities and increase the motility and range of motion of their extremities $(14,15)$.

The purpose of the present study is thus to provide exercises and music that will be helpful for balancing ability, and to determine their effects in order to provide data for the promotion of elderly persons' health.

\section{METHODS}

\section{Subjects}

The present study was conducted with 30 elderly persons living at $\mathrm{H}$ nursing facility in Yongin-si, South Korea. The study subject selection criteria are as follows:

a. Those who have had no experience of falling for the last year;

b. Those who can walk continuously at least $5 \mathrm{~m}$;

c. Those who have no pain that affects walking;

d. Those who do not take any medications that can affect balancing ability;

e. Those who have no neurological or musculoskeletal system abnormalities that may lead to imbalance or falls; f. Those whose score in the Korean version of the Korean version of the mini-mental state examination ( $\mathrm{k}-\mathrm{MMSE})$ is at least 20 points;

g. Those who have serious damage to vision or somesthesia that could affect the experiment.

The subjects' physical features are shown in Table 1.

Table 1. General features of the research subjects

\begin{tabular}{|c|c|c|}
\hline & BTG(Mean士SD) & BTMTG(Mean士SD) \\
\hline Age(y) & $62.53 \pm 4.41$ & $63.01 \pm 3.21$ \\
\hline Height(cm) & $160.07 \pm 5.42$ & $162.54 \pm 2.01$ \\
\hline Weight(kg) & $61.36 \pm 7.83$ & $63.14 \pm 4.01$ \\
\hline MMSE & $26.31 \pm 3.28$ & $26.98 \pm 4.20$ \\
\hline
\end{tabular}

BTG: balance training group; BTMTG: balance training + music therapy group; K-MMSE: Korean version of the mini-mental state examination.

\section{Procedure}

The experimental procedure is as follows. Before participating in each environment, the subjects' physical features and MMSE scores were measured. They were then randomly assigned to a balance training group(BTG) of 15 subjects, or a music and balance training group(music therapy + balance training[MTBTG]) of 15 subjects; interventions were implemented three times per week for six weeks. The content of the experiment was sufficiently explained to the voluntary experimental participants who had been selected, so that they understood the purpose of the experiment. The experiment was conducted only with participants who signed the agreement.

\section{Balance training}

The balance training consisted of exercises performed in standing positions on a hard floor; the exercises were performed using an aero step(19). During the exercises, for ankle strengthening strategies, joint movements were consciously limited by the experimental subject, and the therapist observed and guided the exercises when necessary. Each exercise program(1 set) was 30 minutes; there were also warm up exercises $(5$ minutes) before the exercise program and coolingdown exercises (5 minutes) afterwards. The balance training program is as shown in Table 2. 
The Effects of Music during Balance Training for Six Weeks on Elderly Persons' Balancing Ability

Table 2. Balance exercise program

\begin{tabular}{cc}
\hline EXERCISE PROGRAM & Time \\
Warm up exercise & 5 minutes \\
Exercise performed in a standing position on stable, hard ground \\
Ankle strategy exercise: raising the heels of both feet slowly \\
Ankle strategy exercise: raising the heels of both feet \\
Ankle strategy exercise: standing on alternate feet \\
Aero-step exercise & 5 minutes \\
Ankle joint strategy exercise: maintaining balance in a static posture & 5 minutes \\
Ankle joint strategy: shaking the body in the anterior, posterior, left, & 5 minutes \\
right, and diagonal directions & 10 minutes \\
Cool-down exercise & 5 minutes
\end{tabular}

\section{Music therapy procedure}

The subjects selected the music that they would listen to during balance training; their choice was limited to slower-tempo popular music to ensure consistency. In order to minimize errors from the music, the tempos of the music were collected in advance and measured in beats per minute(BPM).

\section{Timed Up and Go(TUG) test}

The TUG is a test method that can quickly measure basic motility and balance. The test measures the time it takes for a subject sitting in a chair with armrests to get up, walk a distance of $3 \mathrm{~m}$, come back, and sit in the chair again(18). Before measuring, the test method was sufficiently explained and demonstrated by the researcher, and each subject practiced the method 2 3 times.

\section{Berg Balance Scale(BBS)}

The Berg Balance Scale was used in the present study to measure balancing ability. The BBS consists of 14 items that can be roughly divided into three areas: sitting, standing, and posture changes. Scores range from 0 points (the minimum) to 4 points(the maximum); the highest score for balance is 56 points, since there are 14 items. This measuring tool has high intra-rater reliabili$\operatorname{ty}(r=.99)$, inter-rater reliability $(r=.97)$, and internal validity in assessing balance ability(16, 17). Each study subject was measured three times, and the average value was recorded.

\section{Limits of stability(LOS)}

In the present study, a HUR BT4(HUR Labs,
Tampere, Finland) was used for the LOS test. The HUR BT4 platform is an elaborate and portable piece of assessment equipment designed for improved tests of stability. The BT4(HUR Labs) model by Apsun Inc. was used for measurement through the limit of stability of the ankle. Each subject mounted the platform with bare feet and maintained a standing position. The equipment's screen was placed behind the subjects to prevent them from seeing the screen; the subjects tilted their bodies forward, backward, leftward, and rightward using only their ankle joints while maintaining the posture for $8 \mathrm{sec}$. per direction to measure the limit of stability. Each study subject was measured three times, and the average value was recorded. The sensitivity of BT4 is $2(\mathrm{~V} / \mathrm{V} \pm$ $0.25 \%$ ), and the maximum range of error is $0.03 \%$.

\section{Data analysis}

The data measured in the present study were analyzed using the SPSS ver. 18.0 program. The general features were indicated as mean values and standard deviations. Levene $\mathrm{F}$-tests were used to verify the homogeneity of the subjects, and Kolmogorov-Smirnov tests were used to prove the normal distribution. Two-way repeated measure ANOVAs were conducted to compare differences in BBS, LOS, and variables between groups(BTG and BTMTG) and times(before and after intervention). Significant differences between pre-tests and post tests were verified using paired sample t-tests. The statistical significance level was set to $\alpha=.05$. 


\section{RESULTS}

\section{Changes in the limits of stability during balance training}

Changes in the limits of stability before and after the intervention were shown to be significant in both the BTG and the MTBTG(Table 3). Changes in the limits of stability of the right side were shown to be statistically different between the BTG and the MTBTG(Table 3).

Table 3. Comparison in the limits of stability before and after the intervention.

\begin{tabular}{|c|c|c|c|c|c|}
\hline Variable & Group & pre(M $\pm S D)$ & $\operatorname{post}(M \pm S D)$ & & $\mathrm{p}$ \\
\hline \multirow{2}{*}{ Anterior } & BTG & $4.354 \pm .838$ & $5.256 \pm 1.747 \dagger$ & group & .063 \\
\hline & MTBTG & $3.536 \pm .476$ & $4.870 \pm .530 \dagger$ & $\begin{array}{l}\text { time } \\
\text { group*time }\end{array}$ & $\begin{array}{l}.0000^{n+} \\
.314\end{array}$ \\
\hline \multirow{2}{*}{ posterior } & BTG & $3.554 \pm .476$ & $4.076 \pm .791 \dagger$ & group & .037 \\
\hline & MTBTG & $2.746 \pm .793$ & $3.306 \pm .424 \dagger$ & group*time & .371 \\
\hline \multirow[t]{2}{*}{ Left } & BTG & $2.983 \pm 1.570$ & $4.196 \pm 1.023 \dagger$ & group & $\begin{array}{l}.051 \\
.001 *\end{array}$ \\
\hline & MTBTG & $2.074 \pm 1.258$ & $3.200 \pm 1.430 \dagger$ & group*time & .624 \\
\hline \multirow[t]{2}{*}{ Right } & BTG & $2.785 \pm 1.586$ & $3.873 \pm 2.570 \dagger$ & group & $.039^{*}$ \\
\hline & MTBTG & $2.958 \pm .946$ & $4.187 \pm 1.218 \dagger$ & group*time & .003 \\
\hline
\end{tabular}

${ }^{* *} \mathrm{p}\left\langle .001 ;{ }^{*} \mathrm{p}\langle .05 \dagger\right.$ statistically significantly different from pretests; BTG: balance training group; MTBTG: balance training + music therapy group

\section{Changes in the TUG during balance training with} music therapy

Changes in the TUG before and after the intervention were shown to be significant in both the
BTG and the MTBTG(Table 4). In addition, interactions between time and groups were shown to be statistically significant in the two groups(Table 4).

Table 4. Comparison in the TUG before and after the intervention.

\begin{tabular}{|c|c|c|c|c|c|}
\hline Variable & Group & pre(M $\pm S D)$ & $\operatorname{post}(M \pm S D)$ & & $\mathrm{p}$ \\
\hline \multirow{2}{*}{ TUG } & BTG & $13.671 \pm 3.896$ & $10.935 \pm 4.938 \dagger$ & group & .039 \\
\hline & MTBTG & $14.442 \pm 5.711$ & $9.741 \pm 11.054 \dagger$ & group*time & $.000^{* *}$ \\
\hline
\end{tabular}

${ }^{* *}$ p <.001, ${ }^{*} \mathrm{p}<.05 \dagger$ statistically significantly different from pretests; BTG: balance training group, BTMTG: balance training + music therapy group

\section{Changes in BBS during balance training with music therapy}

Changes in the BBS before and after the intervention were shown to be significant in both the
BTG and the MTBTG(Table 5). In addition, interactions between time and groups were shown to be statistically significant in the two groups(Table 5).

Table 5. Comparison in the BBS before and after the intervention.

\begin{tabular}{|c|c|c|c|c|c|}
\hline Variable & Group & $\operatorname{pre}(\mathrm{M} \pm \mathrm{SD})$ & $\operatorname{post}(\mathrm{M} \pm \mathrm{SD})$ & & $\mathrm{p}$ \\
\hline \multirow{2}{*}{ BBS } & BTG & $50.873 \pm 3.734$ & $54.00 \pm 3.873 \dagger$ & group & .058 \\
\hline & MTBTG & $59.328 \pm 2.264$ & $53.24 \pm 2.274 \dagger$ & group*time & $.000^{* *}$ \\
\hline
\end{tabular}

${ }^{* *} p<.001,{ }^{*} \mathrm{p}<.05 \dagger$ statistically significantly different from pretests; BTG: balance training group; BTMTG: balance training + music therapy group 


\section{DISCUSSION}

Preventing falls or physical damage by improving elderly persons' ability to recover their balance through regular exercise is very important. "Posture control" refers to the ability to control the body in space; this ability can be improved through exercises that improve balancing ability(20). Therefore, in the present study, exercises that are helpful for balancing ability were provided to elderly persons together with music; their changes in balancing ability were measured to determine the effects of such exercises on their balancing ability.

In the present study, intragroup changes in the limits of stability before and after the intervention were shown to be significant in both the BTG and the MTBTG.

Music is frequently used in facilities for elderly persons and hospitals to provide a calm and positive atmosphere, because music relaxes tension and helps to stabilize people's balance(21). In addition, music has an effect on psychological and physiological activities related to autonomic nervous system function control(22). A study conducted by Scartelli reported that steady music played by musical instruments had positive effects on the electromyogram biofeedback relaxation training of patients with cerebral palsy due to $\operatorname{spasm}(23)$. Other study results have indicated that breathing rates and heart rates decrease when relaxing music is repeatedly listened to compared to when stimulating music is listened to $(24,25)$. Karlsson et al. reported that elderly persons showed more flexible movements when they were exercising while listening to music of voices and musical instruments than when they were exercising without music(13). As such, when elderly people exercise while listening to music, their autonomic nervous systems cause changes to their muscle responses and cardiovascular system; thus, muscle exercises with and without music show statistically significant differences in changes in the limits of stability between BTG and MTBTG(26, 27).

In the present study, changes in the TUG and BBS tests before and after the experiment were shown to be statistically significant in both the BTG and the MTBTG. In addition, interactions between time and groups were shown to be statistically significant in the two groups.

Pajala et al. compared an ankle balance training group and an ankle muscle strengthening exercise group; the former showed less postural swaying than the latter. Shigematsu et al.(2002) reported that when elderly persons were trained with single leg standing, squatting, marching, and heel touching, as well as targeted balance, strength, and locomotion/agility, three times per week for 12 weeks, the TUG time was shortened. Hwang $\mathrm{Su}-$ Jin reported that when elderly women performed ball exercises five times per week for eight weeks, the subjects' BBS scores improved statistically significantly compared to before the intervention. Other study results have indicated that BBS scores were improved when elderly women participated in muscle strengthening exercises and balance training $(30,31)$. As with the previous studies, in the present study statistically significant improvement in TUG tests and BBS scores appeared in both the BTG and the MTBTG. We attribute this to the balance training that was implemented in both groups, because the training stimulated the lower extremity muscles and trunk muscles, thereby positively affecting balance.

The limitations of the present study are as follows. First, since a large number of subjects could not be included, and the subjects were limited to elderly people hospitalized at a single geriatric hospital, the study results cannot be generalized. Second, because the study was conducted for a short period of time(six weeks), the long-term effects thereafter could not be assessed. Therefore, in future studies, the effects on regional residents for periods of time exceeding six months should be verified.

\section{CONCLUSION}

In the present study, 30 elderly persons were randomly assigned to a balance training group(BTG) of 15 subjects and a music therapy + balance training group(BTMTG) of 15 subjects; interventions were implemented three times per week for six weeks. In order to measure changes in balancing ability, the limit of stability, Timed Up and Go(TUG) test, and the Berg Balance Scale(BBS) were measured. Changes in the limit of stability before and after intervention were shown to be significant in both the BTG and the MTBTG. Changes in the limit of stability of the right side showed statistically significant differences between the BTG and the MTBTG. Changes in the TUG and BBS tests before and after intervention 
were shown to be statistically significant in both the BTG and the MTBTG. The application of music during balance training is thus considered to have positive effects on elderly persons' balancing ability.

\section{REFERENCES}

1. KIm HS. The effect of progressive exercise on the activities of the elderly. Kor Res Soc Phys Ther 2000; 7(3); 1-15.

2. Kang HG, Dingwell JB. Effects of walking speed, strength and range of motion on gait stability in healthy older adults. J Biomech 2008; 41(14): 2899-2905.

3. Kent-Braun JA, Ng AV, Young K. Skeletal muscle contractile and noncontractile components in young and older women and men. J Appl Physiol. 2000; 88(2): 662-668.

4. Goodpaster BH, Carlson CL, Visser M, Kelley DE, Scherzinger A, Harris TB, Stamm E, Newman $A B$. Attenuation of skeletal muscle and strength in the elderly: The Health ABC Study. J Appl Physiol 2001; 90(6): 2157-2165.

5. Schlicht J, Camaione DN, Owen SV. Effect of intense strength training on standing balance, walking speed, and sit-to-stand performance in older adults. J Gerontol A Biol Sci Med Sci 2001; 56(5): M281-286.

6. Sung SC, Kang CG, Lee MG. Effects of falling experience on physical fitness, isokinetic Leg strength, and balance in the elderly women. Kor Alliance Heal Phys Educa Recrea Dance 2007; 46(3); 503-515.

7. Fitzpatrick R, Burke D, Gandevia SC. Loop gain of reflexes controlling human standing measured with the use of postural and vestibular disturbances. J Neurophysiol 1996; 76(6): 3994-4008.

8. Lee SN, The effect of exercise desinged for the aging on health promotion in the rural elderly, Unpublished doctoral dissertation, Yonsei University 1991.

9. Jessup JV, Horne C, Vishen RK, Wheeler D. Effects of exercise on bone density, balance, and self-efficacy in older women. Biol Res Nurs 2003; 4(3): 171-180.

10. Fatouros IG, Taxildaris K, Tokmakidis SP, Kalapotharakos V, Aggelousis N, Athanasopoulos S, Zeeris I, Katrabasas I. The effects of strength training, cardiovascular training and their combination on flexibility of inactive older adults. Int
J Sports Med 2002; 23(2): 112-119.

11. Shin YH, Choi YH. The effect of walking exercise program on cardiorespiratory function and flexibility in elderly women. Kor Soc Nur Sci 1996; 26(2): 372-386.

12. Sun WD, Lee SH, Park JS, Bae SS, Cho YH. Analysis of the effects of muscle strength exercise on physical function and quality of life in the frail elderly. J Kore Soc Heal Educa Promot 2008; 25(1); 39-53.

13. Lee YY, Chan MF, Mok E. Effectiveness of music intervention on the quality of life of older people. J Adv Nurs 2010; 66(12): 2677-2687.

14. Silverman MJ. The effect of pitch, rhythm, and familiarity on working memory and anxiety as measured by digit recall performance. $J$ Music Ther 2010; 47(1): 70-83.

15. Sherratt K, Thornton A, Hatton C. Music interventions for people with dementia: a review of the literature. Aging Ment Health 2004; 8(1): 312.

16. Lee JW. Effect of ankle strategy exercise on improvement of balance in elderly with impaired balance. Unpublished doctoral dissertation, Yonsei University 2007.

17. Podsiadlo D, Richardson S. The timed "Up \& Go": a test of basic functional mobility for frail elderly persons. J Am Geriatr Soc 1991; 39(2): 142-148.

18. Berg KO, Wood-Dauphinee SL, Williams JI, Maki B. Measuring balance in the elderly: validation of an instrument. Can J Public Health 1992; 83 Suppl 2: S7-11.

19. Bogle Thorbahn LD, Newton RA. Use of the Berg Balance Test to predict falls in elderly persons. Phys Ther 1996; 76(6): 576-583.

20. Shumway-Cook A, Woollacott M, Kerns KA, Baldwin. The effects of two types of cognitive tasks on postural stability in older adults with and without a history of falls. J Gerontol A Biol Sci Med Sci 1997; 52(4): M232-240.

21. Munro S, Mount B. Music therapy in palliative care. Can Med Assoc J 1978; 119(9): 1029-1034.

22. McCraty R, Barrios-Choplin B, Atkinson M, Tomasino D. The effects of differenttypes of music on mood, tension, and mental clarity. Altern Ther Health Med 1998; 4(1): 75-84.

23. Scartelli JP. The Effect of Sedative Music on Electromyographic Biofeedback Assisted Relaxation Training of Spastic Cerebral Palsied Adults. J Music Ther 1982; 21: 67-78.

24. Iwaki T, Iwanaga M, Ikeda M. Effect of repetitive exposure of music on subject and physiology responses. J Music Ther 1996; 33: 219-230. 
25. Hammer SE. The effect of guided imagery through music on state and trait anxiety. J Music Ther 1996; 33: 47-30.

26. Davies CT, Few JD. Effects of exercise on adrenocortical function. J Appl Physiol 1973; 35(6): 887-891.

27. Park HO. Structure of the Music Therapy and its Substantiality Research. J smeieccu 2000; 3(1): 151-183

28. Pajala S, Era P, Koskenvuo M, Kaprio J, Tolvanen A, Heikkinen E, Tiainen K, Rantanen $\mathrm{T}$. Contribution of genetic and environmental effects to postural balance in older female twins. J Appl Physiol 2004;96(1): 308-315.
29. Shigematsu R, Chang M, Yabushita N, Sakai T, Nakagaichi M, Nho H, Tanaka K. Dance-based aerobic exercise may improve indices of falling risk in older women. Age Ageing 2002; 31(4): 261-266.

30. Kim TH, Choi HS. Effects of 4 weeks bridging stabilization exercise using swiss ball and whole body vibration on balance and gait function in elderly women. Kor Res Soc Phys Ther 2011; 18(3): 49-58.

31. Englund U, Littbrand H, Sondell A, Pettersson U, Bucht G. A 1-year combined weight-bearing training program is beneficial for bone mineral density and neuromuscular function in older women. Osteoporos Int 2005; 16(9): 1117-1123. 